\title{
Multicystic vestibular schwannomas with fluid-fluid levels: A report of three cases
}

\author{
PUXIAN LI ${ }^{1,2}$, ZHIYONG BI ${ }^{2}$, SHUYU HAO ${ }^{2}$ and JUNTING ZHANG ${ }^{2}$ \\ ${ }^{1}$ Department of Neurosurgery, Laiwu Hospital, Taishan Medical University, Laiwu, Shandong 271100; \\ ${ }^{2}$ Department of Neurosurgery, Beijing Tiantan Hospital, Capital Medical University, Beijing 100050, P.R. China
}

Received August 14, 2014; Accepted April 14, 2015

DOI: $10.3892 / \mathrm{ol} .2015 .3211$

\begin{abstract}
The present study reported three rare cases of multicystic vestibular schwannoma with fluid-fluid levels. These three tumors exhibited apparent fluid-fluid levels that demonstrated a radiological appearance of two fluid levels in the cystic area. Two cases had an acute history, whereas one case presented with a chronic progression. A suboccipital retrosigmoid approach was used for access to the tumors and gross total resection was achieved in all the cases. During the surgical procedures, unclotted blood was observed, and the tumors were adhered to the facial nerve, other cranial nerves or the brain stem. In each case, the facial nerve function of the patients was impaired following surgery. The present study discussed the possible mechanism of the formation of the fluid-fluid levels, the effect of the fluid-fluid levels and the therapeutic strategy employed.
\end{abstract}

\section{Introduction}

Vestibular schwannomas (VSs) are benign, slow-growing neoplasms that develop in the cerebellopontine angle (CPA) area of the brain (1). VSs may be subdivided into cystic and solid according to their morphology $(2,3)$. Cystic VSs are relatively less common than solid VSs, with a reported incidence rate ranging between 5.7 and 24\% (2). Furthermore, cystic VSs are more aggressive than solid VSs due to the rapid growth and unpredictable expansion of their cystic component $(2,3)$.

Fluid-fluid levels in tumors display a radiological appearance of two fluid levels in the cystic section of tumors (4-10). This level is apparent on computed tomography (CT) and magnetic resonance imaging (MRI) scans, particularly on T2-weighted MRI scans (4-11). Previous studies have reported fluid-fluid levels in non-neurogenic tumors $(4,5)$, as well as

Correspondence to: Dr Junting Zhang, Department of Neurosurgery, Beijing Tiantan Hospital, Capital Medical University, 6 Tiantan Xili, Dongcheng, Beijing 100050, P.R. China

E-mail: zjt_ttsw@163.com

Key words: fluid-fluid level, multicystic vestibular schwannoma, vestibular schwannoma a small number of cranial nerve schwannomas, including trigeminal, glossopharyngeal and hypoglossal schwannomas (6-8). However, cystic VSs with fluid-fluid levels are fairly rare $(3,6,9,10)$.

The current study presented three cases of multicystic VS with fluid-fluid levels, introducing their clinical manifestations, imaging features and surgical findings. In addition, the possible mechanism of fluid-fluid level formation, the effect of fluid-fluid levels and the therapeutic strategy employed were discussed. This study was approved by the ethics committees of Laiwu Hospital (Laiwu, Shandong, China) and Beijing Tiantan Hospital (Beijing, China), and written informed consent was obtained from each of the patients.

\section{Case reports}

Case one. In December 2013, a 65-year-old male patient presented to Beijing Tiantan Hospital with a six-month history of right-sided facial numbness and sialorrhea, with no tinnitus, hearing loss, headache or other symptoms. Neurological examination demonstrated impaired sensation to light touch and pinprick testing in the maxillary division of the right trigeminal nerve. The preoperative right-sided facial nerve function was diagnosed as House-Brackmann grade II (11).

CT and MRI scans identified a predominately multicystic mass with two apparent fluid-fluid levels in the right CPA extending to an enlarged internal acoustic meatus, measuring $4.3 \times 2.9 \times 3.3 \mathrm{~cm}$ (Fig. 1). The right cerebellum and brainstem were markedly distorted by the lesion; however, the fourth ventricle was not clearly compressed and there was no evidence of hydrocephalus.

Microsurgery was performed using a suboccipital retrosigmoid approach (12) with intraoperative neurophysiological monitoring and gross-total resection was achieved. During the surgical procedure, a yellow-green fluid and unclotted blood were observed in the cyst of the tumor. Furthermore, the tumor adhered to the brainstem and facial nerve. The brainstem was intact following tumor resection and the facial nerve was anatomically preserved. Histopathological findings revealed that the tumor was hypercellular and composed of spindle-shaped cells with obvious palisade arrangement and regular nuclei, which indicated a diagnosis of VS with Antoni type A. Postoperatively, the patient developed House-Brackman grade $\mathrm{V}$ facial nerve palsy and hearing loss, 
A

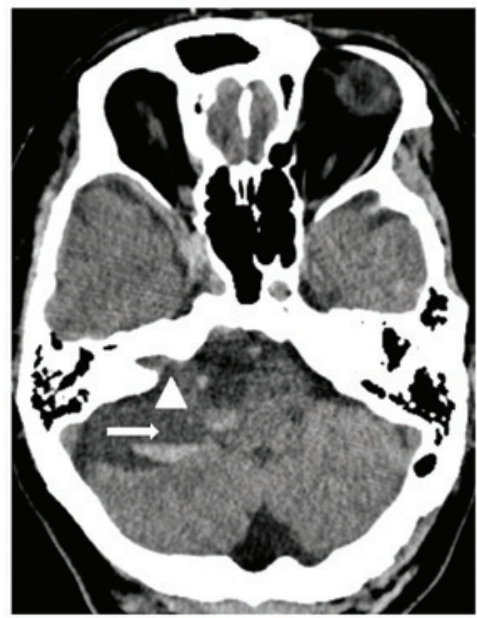

D

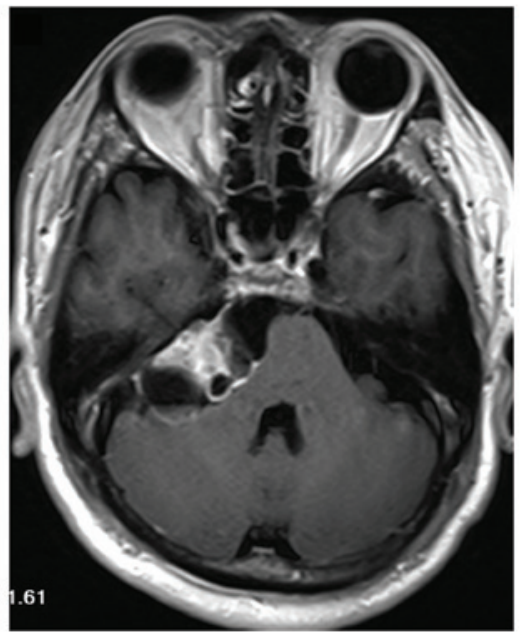

B

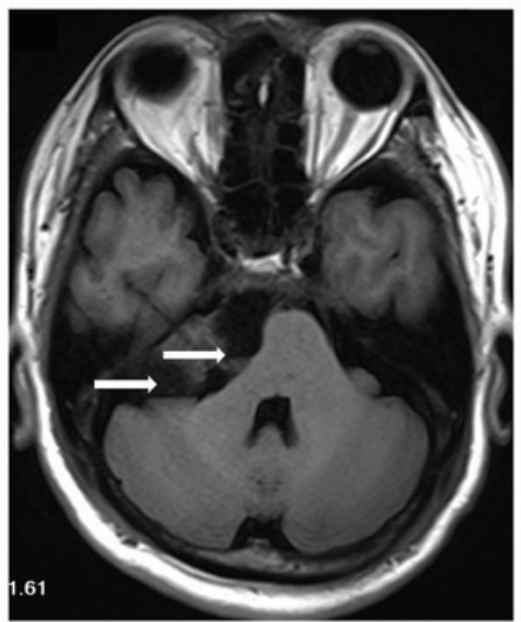

E

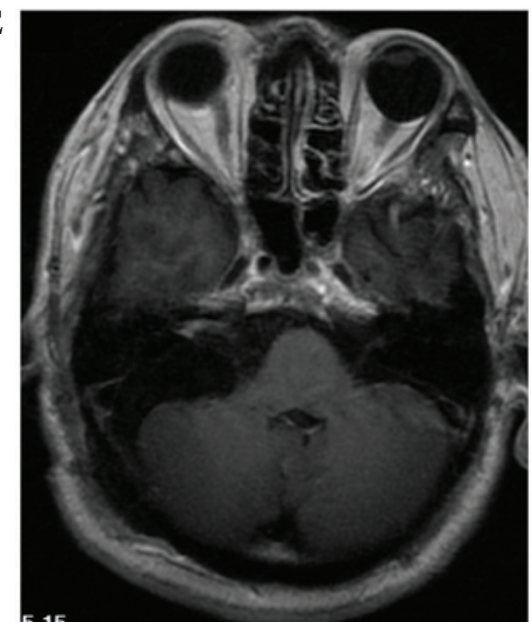

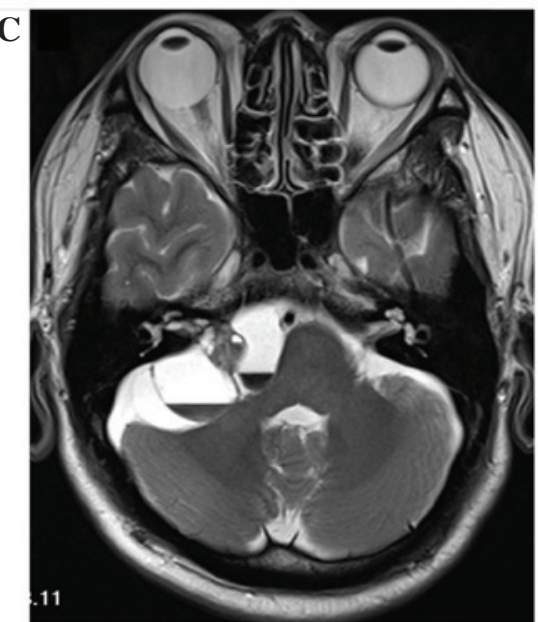

F

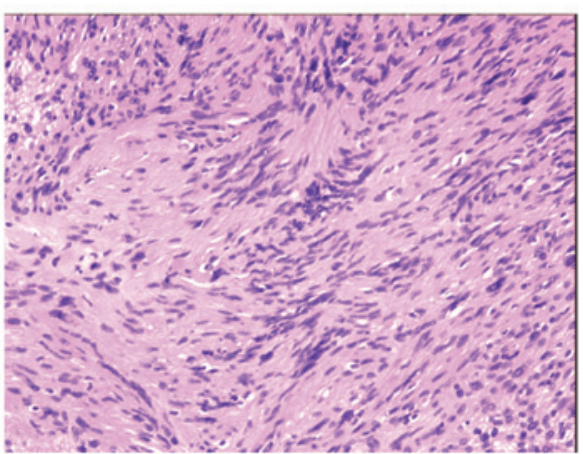

Figure 1. Preoperative and postoperative images of case one. (A) Axial CT scan revealing a mass in the right cerebellopontine angle. Arrow indicates the fluid-fluid level (the superior layer being of a lower density and the inferior layer of a higher density). Arrow head indicates the enlarged internal acoustic meatus. (B) Axial T1-weighted MRI scan, identifying two apparent fluid-fluid levels (arrows) in the multicystic mass. The inferior layer and the solid section of the mass produced an isointense signal, while the superior layer produced a hypointense signal. Furthermore, the mass compressed the cerebellum and the brainstem, extending into the internal acoustic meatus. (C) Axial T2-weighted MRI scan, revealing a hyperintense signal from the superior layer of the fluid-fluid level and a hypointense signal from the inferior layer. The basilar artery, and the left seventh and eighth cranial nerves are distinctly visible in this image. (D) Axial T1-weighted enhanced MRI scan, revealing enhancement in the capsule and the solid section of the tumor, including the meatal component. (E) Postoperative axial T1-weighted enhanced MRI scan, demonstrating that the tumor was completely resected during surgery. (F) Histological analysis, indicating that the tumor has characteristics consistent with schwannoma (hematoxylin and eosin staining; original magnification, x100). CT, computed tomography; MRI, magnetic resonance imaging.

which did not resolve over the six-month follow-up period. Hypoglossal-facial nerve anastomosis was proposed; however, the patient did not consent to the procedure. The tumor did not recur during the six-month follow-up period.

Case two. A 59-year-old female patient was admitted to Laiwu Hospital in March 2014 with a 1.5-year history of worsening hearing loss in the left ear, headache, dizziness, a four-month history of left-sided facial numbness, coughing when consuming liquids, dysphagia and an unsteady gait. Two months prior to admission, the aforementioned symptoms had suddenly worsened, and the patient experienced a severe headache and was unable to walk due to ataxia. Physical examination revealed impaired sensation in the maxillary and mandibular divisions of the left trigeminal nerve, ataxia and dysmetria on the finger-nose test. The pure-tone average in the left ear was determined to be $45 \mathrm{~dB}$. The pure-tone thresholds were determined for the left and right ears using the modified
Hughson-Westlake method at frequencies of $0.25,0.50,1.0$, $2.0,4.0$ and $8.0 \mathrm{kHz}(13)$. In addition to assessing absolute hearing values, clinical function was categorized into the following hearing loss (HL) ranges: $<20 \mathrm{~dB}$, normal hearing; 20-40 dB, mild HL; 40-60 dB, moderate HL; 60-70 dB, moderately severe HL; 70-90 dB, severe HL; and $>90 \mathrm{~dB}$, profound HL.

MRI scans revealed a multicystic lesion (Fig. 2) with two apparent fluid-fluid levels in the left cerebellopontine area. The mass, which measured $4.6 \times 3.4 \times 3.4 \mathrm{~cm}$, compressed the left cerebellum, the brainstem and the fourth ventricle, forming a cerebellar tonsil hernia with no hydrocephalus. Prior to surgery, the House-Brackmann grade was determined to be I (normal).

The tumor was completely resected using a retrosigmoid approach. The cystic fluid was yellow-green in color with unclotted blood, and the solid section was yellow-white. The tumor arose from the eighth cranial nerve and was 

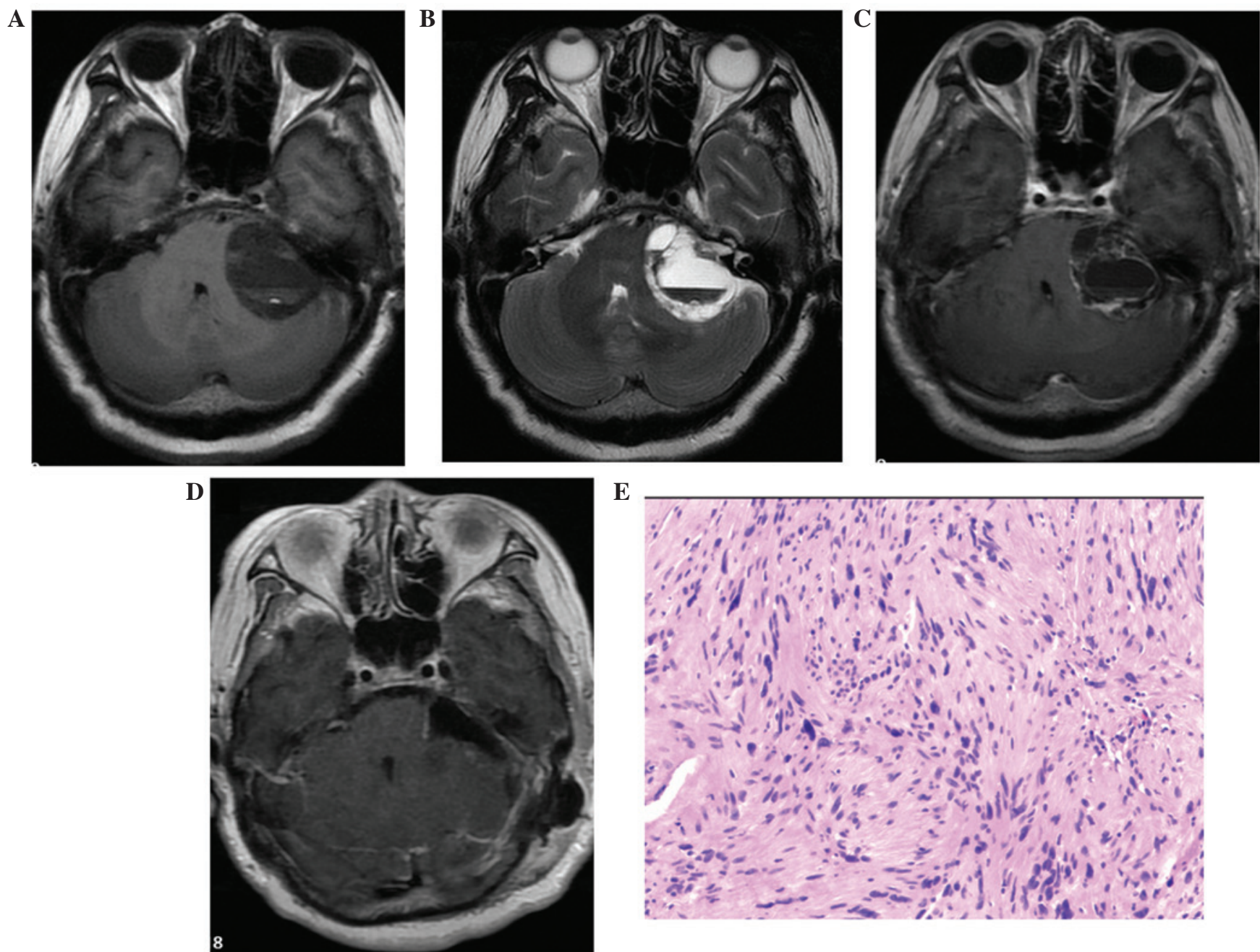

Figure 2. Preoperative and postoperative images of case two. (A) Axial T1-weighted MRI scan, revealing that the mass predominantly consists of iso- and hypointense components in the left cerebellopontine angle. The left cerebellum, brainstem and fourth ventricle were compressed. (B) Axial T2-weighted MRI scan, demonstrating that the tumor was composed of numerous cysts. In addition, two apparent fluid-fluid levels can be observed. (C) Axial T1-weighted enhanced MRI scan, revealing a solid portion and the capsule of the cyst exhibiting enhancement. (D) Postoperative axial T1-weighted enhanced MRI scan, demonstrating that the tumor was completely resected. (E) Histopathological examination clarifying that the tumor was a schwannoma (hematoxylin and eosin staining; original magnification, x100). MRI, magnetic resonance imaging.

compressing and adherent to cranial nerves X, XI and XII, as well as the lower cranial nerves, which were carefully dissected from the tumor under intraoperative neurophysiological monitoring. Histological examination indicated that the tumor was composed of spindle-shaped cells with palisade arrangement (Antoni type A) and some nuclei were enlarged and darkly stained; a diagnosis of VS was therefore determined. The patient developed House-Brackmann grade III facial nerve palsy and had no effective hearing on the left side during the three-month follow-up period. No tumor recurrence was observed.

Case three. A 27-year-old male patient presented to Beijing Tiantan Hospital in March 2014 with right-sided tinnitus and hearing loss that lasted for $\sim 1.5$ years, and a six-month history of intermittent dizziness, nausea, vomiting and an unsteady gait. During the month prior to admission, the aforementioned symptoms suddenly worsened. In addition, right-sided facial numbness and limb shaking developed, and the patient was unable to walk due to ataxia. Physical examination revealed impaired sensation in the maxillary division of the left trigeminal nerve, as well as ataxia. The pure tone average in the left ear was determined to be $95 \mathrm{~dB}$.

CT and MRI scans revealed a multicystic tumor with one fluid-fluid level in the left CPA area (Fig. 3). The tumor measured $6.3 \times 4.2 \times 5.2 \mathrm{~cm}$, compressed the right cerebellum, the brainstem and the fourth ventricle, and hydrocephalus was observed. The axial and sagittal images identified a high-density matter in the inferior layer.

The patient underwent surgery using the retrosigmoid approach to completely resect the tumor. The tumor was predominantly composed of cysts in which xanthochromic fluid and unclotted blood were observed. Subsequent histological analysis indicated features characteristic of a schwannoma; most of the area was focal cellular (Antoni A), and part was hypocellular with vacuolar degeneration (Antoni B). Following surgery, the patient developed House-Brackmann grade VI facial nerve palsy and had no effective hearing on the right side during the three-month follow-up period. Tumor recurrence was not observed. 
A

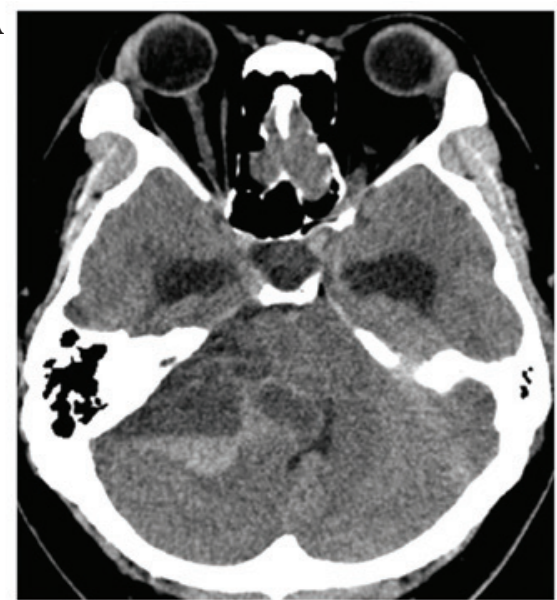

D

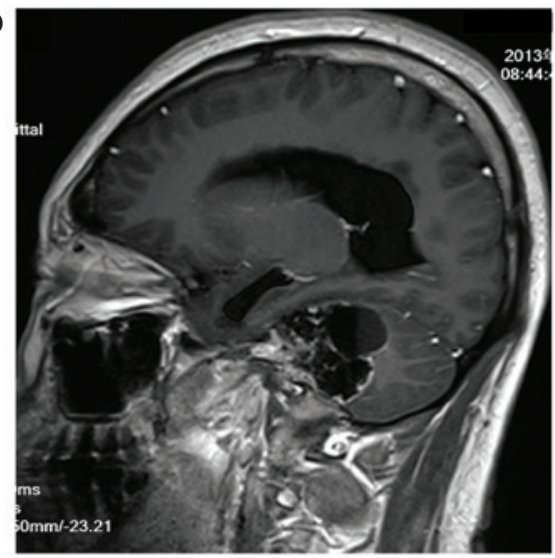

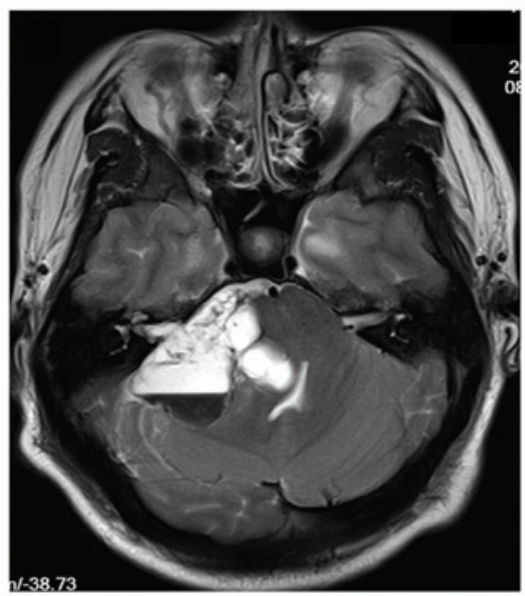
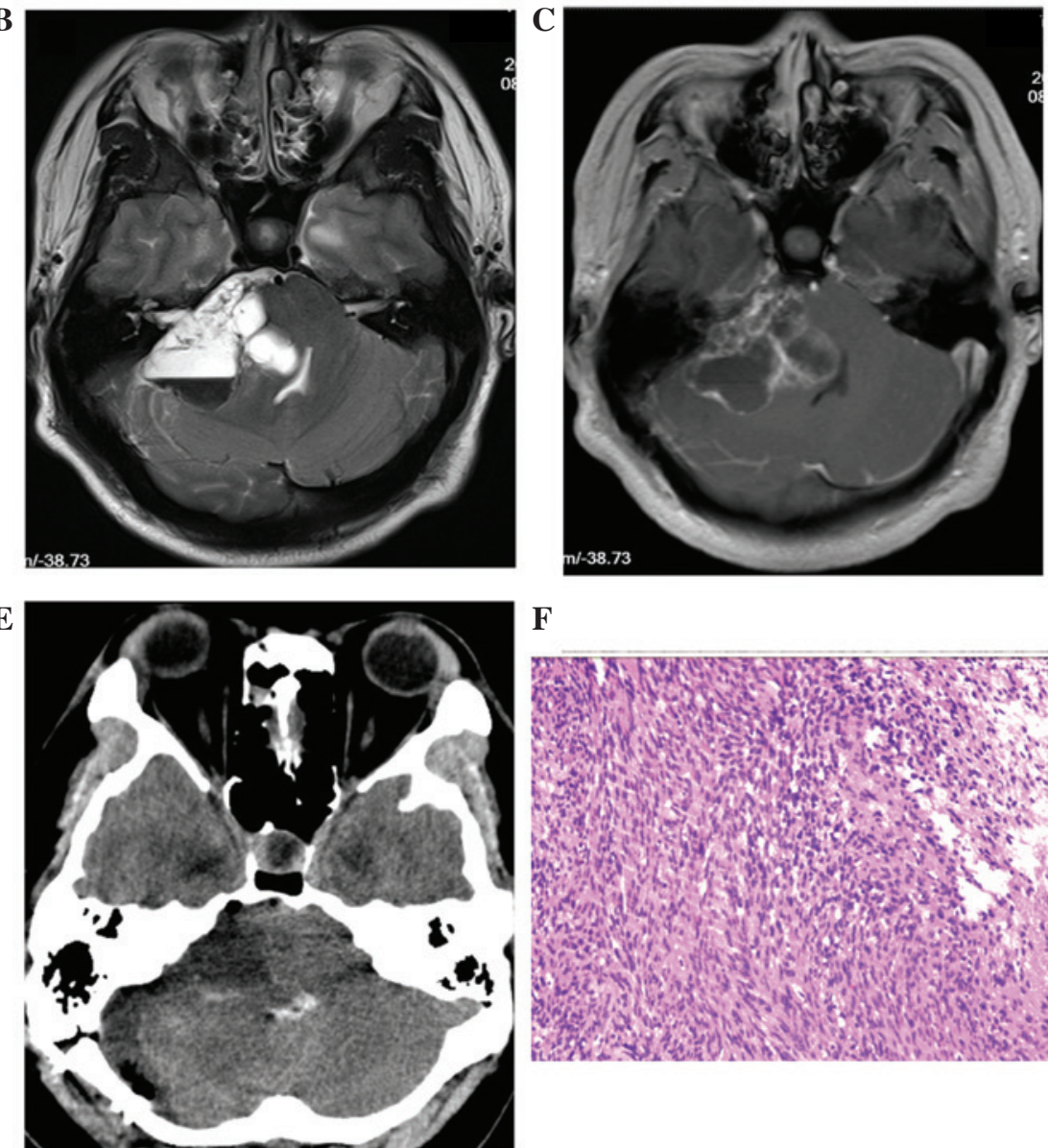

F

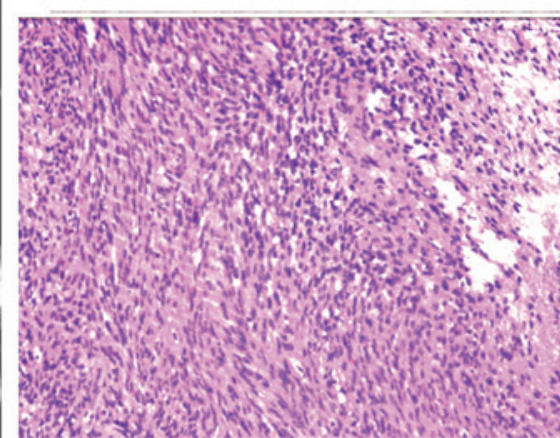

Figure 3. Preoperative and postoperative images of case three. (A) Axial CT scan indicating a lesion located in the right cerebellopontine angle. The lesion consisted of a higher density substance and a lower density substance. (B) Axial T2-weighted MRI scan, demonstrating that the tumor was composed of numerous cysts with apparent fluid-fluid levels. (C) Axial T1-weighted enhanced MRI scan, revealing a solid portion and cyst capsules demonstrating enhancement. (D) Sagittal T1-weighted enhanced MRI scan, revealing a fluid-fluid level parallel to the cranio-caudal axis, due to the gravitational effect of the patient lying in the supine position. (E) Postoperative CT scan showed the tumor was completely resected and a small hemorrhage in the fourth ventricle. (F) Histopathological examination clarifying that the tumor was a schwannoma (hematoxylin and eosin staining; original magnification, x100). CT, computed tomography; MRI, magnetic resonance imaging.

\section{Discussion}

A fluid-fluid level is considered to be an uncommon and non-specific phenomenon in tumors (4). To date, there is no consensus regarding the mechanism underlying the formation of these levels; however, two mechanisms have been proposed in the literature $(2,4,6-8,10,14)$. The first refers to tumor necrosis causing liquefaction and exudation of the tumor tissue. The fluid formed initially is more proteinaceous compared with the interstitial fluid formed later; thus, fluid separation occurs based on viscosity and protein content $(7,10)$. The second proposed mechanism is hemorrhage, typically of unclotted blood. The red blood cells or the products of red blood cell breakdown constitute the inferior fluid layer, while serous blood constitutes the superior fluid layer based upon sedimentation $(2,4,6,8,14)$. The present authors consider the second mechanism to be more accurate, since unclotted blood was observed in all the cases reported in the current study. Furthermore, the growing tumor is hypothesized to compress and erode internal blood vessels, resulting in occlusion, thrombus, ischemia and the elastolytic function of enzymes. Thus, tumor growth may result in the destruction and degeneration of blood vessels, including the formation of pseudoaneurysms and subsequent bleeding (2-4). Due to the different densities of blood cells or blood cell breakdown products and serum, the fluid-fluid levels are proposed to form through the sedimentation effect. In addition, inflammatory exudation, induced by blood breakdown products, possibly occurred during the formation of the layers. Acute hemorrhage within the tumor was considered to be the cause of the sudden worsening of symptoms in cases two and three of the current study (9). By contrast, in case one, which presented with chronic evolution of the condition, microhemorrhages may have occurred.

The presence of fluid-fluid levels can be clarified by performing CT or MRI scans. On CT scans, the superior fluid layer had a lower density, whereas the inferior fluid layer had a higher density, compared with brain tissue (Figs. 1A and 3A). On T1-weighted MRI scans, the superior fluid layer was hypointense and the inferior fluid layer was isointense (Figs. 1B and 2A). Furthermore, on T2-weighted MRI scans, the superior fluid layer presented marked hyperintensity, while the inferior fluid layer was hypointense (Figs. 1C, 2B and 3B).

A number of studies have investigated the significance of fluid-fluid levels in tumors. For instance, Sommer et al (5) 
proposed that fluid-fluid levels in hepatic metastases are a characteristic indication of metastases of neuroendocrine origin. In addition, Xia et al (3) determined that fluid-fluid levels in VSs were a predictor of peritumoral adhesion and are associated with a less favorable surgical outcome. In agreement with this, VS with fluid-fluid levels adhered to the facial nerve, other cranial nerves or the brain stem were observed during the surgical procedures performed in the current cases. However, determination of the fundamental causes and implications of fluid-fluid levels using biochemistry may be required.

VSs with fluid-fluid levels are not appropriate for 'watch and wait' treatment approaches due to the relatively high probability of sudden tumor enlargement due to hemorrhage $(3,5,15)$. Furthermore, radiosurgery is not recommended, as the expansion of the cystic components and possible hemorrhage following radiosurgery may result in sudden deterioration $(2,16,17)$. Therefore, surgery is the optimal treatment strategy for such patients. Although cystic VSs with fluid-fluid levels indicate greater adhesiveness, it is essential that total resection is performed during the initial surgical procedure due to the tendency for accelerated regrowth of residual cystic schwannoma $(8,18)$.

In conclusion, the current study reported three rare cases of multicystic VS with fluid-fluid levels. Fluid-fluid levels in VSs are predominantly identified on CT or MRI scans. Hemorrhage in multicystic VSs may be the major mechanism of fluid-fluid level formation, with acute hemorrhage resulting in sudden deterioration of the patient's clinical condition and microhemorrhages resulting in chronic evolution of the patient's condition. Furthermore, fluid-fluid levels in VSs indicate greater adhesiveness and a poorer prognosis. Therefore, 'watch and wait' approaches or radiosurgery are not appropriate; instead, surgery is recommended as the optimal treatment strategy.

\section{References}

1. Arthurs BJ, Lamoreaux WT, Giddings NA, Fairbanks RK, Mackay AR, Demakas JJ, Cooke BS and Lee CM: Gamma Knife radiosurgery for vestibular schwannoma: case report and review of the literature. World J Surg Oncol 7: 100, 2009.
2. Park CK, Kim DC, Park SH, Kim JE, Paek SH, et al: Microhemorrhage, a possible mechanism for cyst formation in vestibular schwannomas. J Neurosurg 105: 576-580, 2006.

3. Xia L, Zhang H, Yu C, Zhang M, Ren M, Qu Y, et al: Fluid-fluid level in cystic vestibular schwannoma: A predictor of peritumoral adhesion. J Neurosurg 120: 197-206, 2014.

4. $\mathrm{Lu} \mathrm{ZH}$ and $\mathrm{Wu} \mathrm{M}$ : Unusual features in an adult pancreatic hemangioma: CT and MRI demonstration. Korean J Radiol 14: 781-785, 2013.

5. Sommer WH, Zech CJ, Bamberg F, Auernhammer CJ, Helck A, Paprottka PM, et al: Fluid-fluid level in hepatic metastases: A characteristic sign of metastases of neuroendocrine origin. Eur J Radiol 81: 2127-2132, 2012.

6. Fu H, Hao SY, Jia GJ, Zhang JT and Zhang LW: A cystic vestibular schwannoma with a fluid-fluid level. Chin Med J (Engl) 125: 3920, 2012.

7. Catalano P, Fang-Hui E and Som PM: Fluid-fluid levels in benign neurogenic tumors. AJNR Am J Neuroradiol 18: 385-387, 1997.

8. Li WC, Hong XY, Wang LP, Ge PF, Fu SL and Luo YN: Large cystic hypoglossal schwannoma with fluid-fluid level: A case report. Skull Base 20: 193-197, 2010.

9. Gagliardo C, Martines F, Bencivinni F,La Tona G, Lo Casto A and Midiri M: Intratumoral haemorrhage causing an unusual clinical presentation of a vestibular schwannoma. Neuroradiol J 26: 30-34, 2013.

10. Chin KF, Babar J, Tzifa K, Chavda SV and Irving RM: Vestibular schwannomas with fluid-fluid level. J Laryngol Otol 121: 902-906, 2007.

11. House JW and Brackmann DE: Facial nerve grading system. Otolaryngol Head Neck Surg 93: 146-147, 1985.

12. Yamakami I, Uchino Y, Kobayashi E, Yamaura A and Oka N: Removal of large acoustic neurinomas (vestibular schwannomas) by the retrosigmoid approach with no mortality and minimal morbidity: J Neurol Neurosurg Psychiatry 75: 453-458, 2004.

13. Carhart $R$ and Jerger J: Preferred method for clinical determination of pure-tone thresholds. J Speech Hear Disord 24: 330-345, 1959 .

14. Chang WC, Huang GS, Lee HS, Lee CH and Hsu YC: Fluid-fluid level in peripheral nerve schwannoma: Report of a case with histological correlation. Clin Imaging 33: 248-251, 2009.

15. Sinha S and Sharma BS: Cystic acoustic neuromas: Surgical outcome in a series of 58 patients. J Clin Neurosci 15: 511-515, 2008.

16. de Ipolyi AR, Yang I, Buckley A, Barbaro NM, Cheung SW and Parsa AT: Fluctuating response of a cystic vestibular schwannoma to radiosurgery: Case report. Neurosurgery 62: E1164-E1165, 2008.

17. Ganslandt O, Fahrig A and Strauss C: Hemorrhage into cystic vestibular schwannoma following stereotactic radiation therapy. Zentralbl Neurochir 69: 204-206, 2008.

18. Kameyama S, Tanaka R, Honda Y, Hasegawa A, Yamazaki H and Kawaguchi $\mathrm{T}$ : The long-term growth rate of residual acoustic neurinomas. Acta Neurochir (Wien) 129: 127-130, 1994. 